\title{
Use of splenic ultrasound: a new wave for immune thrombocytopenic purpura
}

Paul Cervi, Audrey Murdock, David Rees, Steve Garner, David Grant, Stephen Wright, Mary Dyson
Watford General

Hospital, Vicarage

Road, Watford

P Cervi

$S$ Wright

Whittington Hospital, Highgate Hill, London N19

A Murdock

D Rees

D Grant

United Medical and

Dental Schools of

Guy's and St

Thomas's Hospitals,

Guy's Hospital

Campus, London

Bridge, London

$M$ Dyson

North London Blood

Transfusion Centre,

Colindale, London

$S$ Garner

Correspondence to:

Dr Paul Cervi, Department

of Haematology,

Whittington Hospital,

London N19.

Accepted for publication

25 November 1993

\begin{abstract}
Aims-To examine whether a therapeutic dose of ultrasound waves, when directed through the thoracic wall to the spleen, would significantly affect the platelet count in patients with stable immune thrombocytopenic purpura (ITP).

Methods-Continuous ultrasound at $1 \mathrm{~W} / \mathrm{cm}^{2}$ spatial average-time average (SATA) intensity for up to one minute/ $5 \mathrm{~cm}^{2}$ treatment field was well tolerated in 13 patients with ITP and one with nonHodgkin's lymphoma. Five healthy controls were also similarly treated. Peak platelet increments occurred four to eight hours after ultrasound treatment in the ITP group ( $n=16$ treatments).

Results-The mean peak platelet increment was $6.25 \times 10^{9} / 1$ with a $5 \%$ confidence interval of the mean $(95 \% \mathrm{CI})$ of 3.32 to $8.93 \times 10^{9} / 1(p=0.0004)$. The mean peak platelet increment of normal controls was $6.6(\mathrm{n}=5 ; 95 \% \mathrm{CI}=-2 \cdot 3$ to $15 \cdot 5$; $p=0.21$ ) and for sham treated patients it was $0.66(n=11 ; 95 \% \mathrm{CI}=-1 \cdot 5$ to $2 \cdot 8$; $p=0 \cdot 60)$. There was a significant inverse correlation between patient age in the ITP group and peak platelet increment $(r=-0.60 ; p=0.015)$.
\end{abstract}

Conclusions-Splenic ultrasound is a novel approach to the treatment of ITP, and may find a place in its diagnosis or management.

\section{(F Clin Pathol 1994;47:414-417)}

Splenic macrophages are important effector cells in the sequestration and destruction of antibody coated platelets in immune thrombocytopenic purpura (ITP). This is shown most clearly by spleen removal, which induces an early and dramatic rise in the platelet count in most patients. Other treatments for ITP are unsatisfactory because they have substantial side effects, are expensive, or are slow to act.

Ultrasound waves have been used successfully for many years to treat localised, painful, and inflammatory disorders, and have an extremely good safety record. ${ }^{1}$ Some of the biological effect of ultrasound is mediated directly by its thermal effect on tissues. At thermal and subthermal intensities, ultrasound waves induce the formation of microbubbles within blood and other tissues and it is thought that this "cavitation effect" damages the integrity and function of cell membranes and intracellular organelles. ${ }^{2-6}$
Interference with macrophage function in particular is believed to be of importance in mediating the beneficial effect of ultrasound in inflammatory conditions.

Previous investigators have shown that ultrasound has significant bioeffects on the immune system ${ }^{7}$ and the mononuclear phagocytic system. ${ }^{8-10}$ Saad and Williams ${ }^{11}$ showed that therapeutic ultrasound at a similar intensity to that used in this study, applied over the umbilical region of rats, caused a short lived ( $<30$ minutes) reduced clearance of ${ }^{99 \mathrm{~m}} \mathrm{Tc}$-labelled colloid from the blood. The magnitude of this effect was directly proportional to the product of the intensity and duration of ultrasound treatment.

\section{Methods}

Patients with thrombocytopenia included thirteen with ITP and one patient with hypersplenism secondary to non-Hodgkin's lymphoma (NHL) (Case 7) (table 1). All patients had had previous bone marrow aspirate examinations that showed normal or increased numbers of megakaryocytes. Recently diagnosed patients, pregnant patients, or those with unstable platelet counts (such as those recently started on drug treatment) were excluded. Healthy controls comprised healthy, volunteer members of the laboratory staff.

Patients were asked to rotate about $60^{\circ}$ on to their right-hand side from a supine position and to place their left hand behind their head. The spleen was then localised with conventional diagnostic ultrasound and the longitudinal and transverse diameters of the spleen were measured; the product gave an estimate of splenic surface area. The overlying skin was marked with a skin pencil to show the ultrasonographic window of access to the organ. The subjects remained in the position described, for ultrasound treatment.

An ENRAF Nonius portable ultrasound machine (Sonopuls 463) operating at $1 \mathrm{MHz}$ with a $5 \mathrm{~cm}^{2}$ ERA treatment head was used. Calibration had been carried out previously according to the method of Young and Dyson. ${ }^{12}$ Continuous ultrasound with a spatial average-time average (SATA) intensity of $1 \mathrm{~W} / \mathrm{cm}^{2}$ was applied for up to one minute per $5 \mathrm{~cm}^{2}$ unit of spleen surface area with a maximum treatment time of 12 minutes. Ultrasound was applied to the spleen through the predefined window of access with a high quality coupling medium (Therasonic, EMS). Treatment was interrupted every four minutes 
Table 1 Subject details and platelet responses

\begin{tabular}{|c|c|c|c|c|c|c|c|}
\hline Case No & $\begin{array}{c}\text { Age } \\
(y)\end{array}$ & Sex & Group & $\begin{array}{l}\text { Spleen } \\
\text { diameter } \\
(\mathrm{cm})\end{array}$ & $\begin{array}{l}\text { Treatment } \\
\text { time } \\
\text { (min) }\end{array}$ & $\begin{array}{l}\text { Baseline } \\
\text { platelets } \\
\left(10^{\circ} / l\right)\end{array}$ & $\begin{array}{l}\text { Peak } \\
\text { platelets } \\
\left(10^{\circ} / l\right)\end{array}$ \\
\hline $\begin{array}{l}1 \\
2 \\
3 \\
4 \\
5 \\
6 \\
7 \\
8 \\
9 \\
10 \\
11 \\
12 \\
13 \\
14 \\
15 \\
16-1 \\
16-2 \\
16-3 \\
17 \\
18 \\
19 \\
20 \\
21 \\
8 \\
9 \\
15 \\
16-1 \\
16-2 \\
16-3 \\
17 \\
18 \\
19\end{array}$ & $\begin{array}{l}29 \\
32 \\
27 \\
30 \\
28 \\
76 \\
72 \\
76 \\
52 \\
39 \\
30 \\
28 \\
42 \\
32 \\
75 \\
69 \\
69 \\
69 \\
57 \\
64 \\
71 \\
73 \\
45 \\
76 \\
52 \\
75 \\
69 \\
69 \\
69 \\
57 \\
64 \\
71\end{array}$ & $\begin{array}{l}F \\
M \\
M \\
F \\
M \\
F \\
M \\
F \\
F \\
F \\
M \\
F \\
F \\
F \\
M \\
F \\
F \\
F \\
M \\
F \\
M \\
F \\
M \\
F \\
F \\
M \\
F \\
F \\
F \\
M \\
F \\
M\end{array}$ & $\begin{array}{l}\text { CONTROL-US } \\
\text { CONTROL-US } \\
\text { CONTROL-US } \\
\text { CONTROL-US } \\
\text { CONTROL-US } \\
\text { ITP-US } \\
\text { NHL-US } \\
\text { ITP-US } \\
\text { ITP-US } \\
\text { ITP-US } \\
\text { ITP-US } \\
\text { ITP-US } \\
\text { ITP-US } \\
\text { ITP-US } \\
\text { ITP-US } \\
\text { ITP-US } \\
\text { ITP-US } \\
\text { ITP-US } \\
\text { ITP-US } \\
\text { ITP-US } \\
\text { ITP-US } \\
\text { ITP-SHAM } \\
\text { ITP-SHAM } \\
\text { ITP-SHAM } \\
\text { ITP-SHAM } \\
\text { ITP-SHAM } \\
\text { ITP-SHAM } \\
\text { ITP-SHAM } \\
\text { ITP-SHAM } \\
\text { ITP-SHAM } \\
\text { ITP-SHAM } \\
\text { ITP-SHAM }\end{array}$ & $\begin{array}{c}8 \cdot 8 \\
8 \cdot 7 \\
7 \cdot 8 \\
7 \cdot 3 \\
9 \cdot 7 \\
7 \cdot 4 \\
11 \cdot 8 \\
7 \cdot 5 \\
10 \cdot 5 \\
9 \cdot 9 \\
9 \cdot 6 \\
8 \cdot 1 \\
10 \cdot 9 \\
8 \cdot 2 \\
12 \\
10 \cdot 5 \\
10 \cdot 5 \\
10 \cdot 5 \\
10 \\
4 \\
12 \\
8 \\
9 \\
7 \cdot 5 \\
10 \cdot 5 \\
12 \\
10 \cdot 5 \\
10 \cdot 5 \\
10 \cdot 5 \\
10 \\
4 \\
12\end{array}$ & $\begin{array}{c}6 \\
6 \\
5 \\
5 \\
8 \\
2 \cdot 5 \\
5 \cdot 5 \\
8 \\
10 \\
5 \cdot 5 \\
3 \\
2 \\
2 \cdot 5 \\
4 \cdot 6 \\
12 \\
10 \\
10 \\
10 \\
12 \\
4 \\
12 \\
0 \\
0 \\
0 \\
0 \\
0 \\
0 \\
0 \\
0 \\
0 \\
0 \\
0\end{array}$ & $\begin{array}{r}320 \\
321 \\
185 \\
207 \\
179 \\
21 \\
134 \\
20 \\
131 \\
55 \\
44 \\
61 \\
51 \\
32 \\
57 \\
10 \\
6 \\
8 \\
35 \\
115 \\
46 \\
62 \\
74 \\
16 \\
125 \\
66 \\
6 \\
8 \\
6 \\
33 \\
121 \\
42\end{array}$ & $\begin{array}{r}319 \\
332 \\
194 \\
222 \\
178 \\
22 \\
134 \\
20 \\
132 \\
63 \\
56 \\
74 \\
71 \\
35 \\
61 \\
15 \\
15 \\
12 \\
43 \\
124 \\
49 \\
59 \\
79 \\
20 \\
126 \\
61 \\
6 \\
6 \\
6 \\
33 \\
125 \\
42\end{array}$ \\
\hline
\end{tabular}

with a two minute break to limit the thermal effect. Whenever possible, patients were sham treated on day 1 and treated with ultrasound on day 2 in a single blind fashion.

Full blood count analysis (Technicon H2) was performed before and at several intervals up to 24 hours after the procedure. Instruments were primed and calibrated with the Technicon $H$ Systems SETpoint calibrator before use and platelet counts were derived from the mean of three assays. The coefficient of variation (CV) of platelet counting was established as less than $8 \%$ for platelet counts in the range $15-450 \times 10^{\%} / 1$. There was no significant deterioration in repeatability of platelet counting for samples with platelet counts of less than $15 \times 10^{9} / 1$ (data not shown).

\section{Results}

Splenic ultrasound treatment was well tolerated by all subjects and the planned treatment course was completed in all cases. Four subjects experienced a local sensation of mild heat but in no case did the skin surface temperature exceed $35^{\circ} \mathrm{C}$ (baseline skin temperature range $25-28^{\circ} \mathrm{C}$ ).

Peak platelet increments occurred four to eight hours after ultrasound treatment (table 2). Only the ITP group (ITP-US) showed a significant mean peak platelet increment. Although a similar mean peak platelet increment was found in the control group

Table 2 Group platelet responses ( $\left.x 10^{9} / l\right)$

\begin{tabular}{lclllll}
\hline Group & $n$ & $M P P I$ & $S D$ & Range & $(95 \%$ CI $)$ & $p$ Value \\
\hline ITP-US+NHL-US & 16 & 6.25 & 5.5 & 0 to 20 & $(3.3$ to $8 \cdot 9)$ & 0.0004 \\
ITP-SHAM & 11 & 0.66 & 3.3 & -2 to 5 & $(-1.5$ to $2 \cdot 8)$ & 0.6 \\
CONTROL-US & 5 & 6.6 & $7 \cdot 2$ & -20 to 30 & $(-2.3$ to $15 \cdot 5)$ & $0 \cdot 21$ \\
\hline
\end{tabular}

$\mathrm{n}=$ Number of treatments; MPPI = mean peak platelet increment
(CONTROL-US), this was not significant and became even less evident if the mean peak platelet increment was expressed as a percentage of the baseline platelet count for each patient (fig 1). There was no significant mean peak platelet increment among the sham treated group (ITP-SHAM).
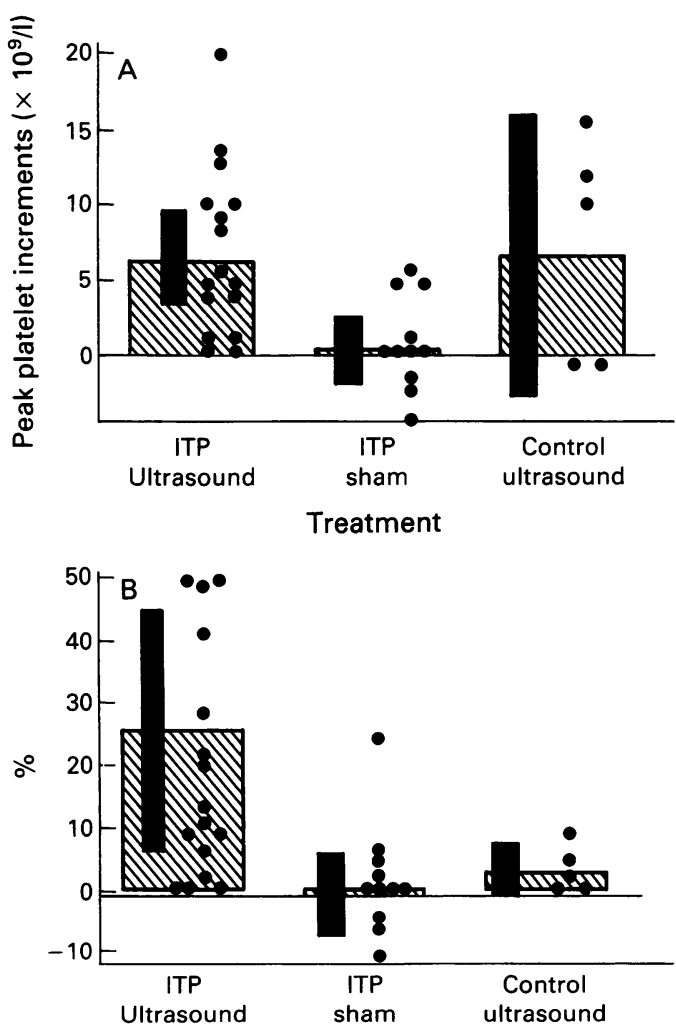

Treatment

Figure 1 Mean peak platelet increment measured four to eight hours after ultrasound or sham treatment expressed as $(A)$ the absolute increment $\left(\times 10^{\circ} / l\right)$ and $(B)$ percentage of the individual baseline platelet count. Solid circles represent individual values. Solid bars represent $95 \%$ CI. 
Figure 2 Simple regression analysis of peak platelet increment in the ITP-ultrasound treated group against patient age $(r=-0 \cdot 60)$.

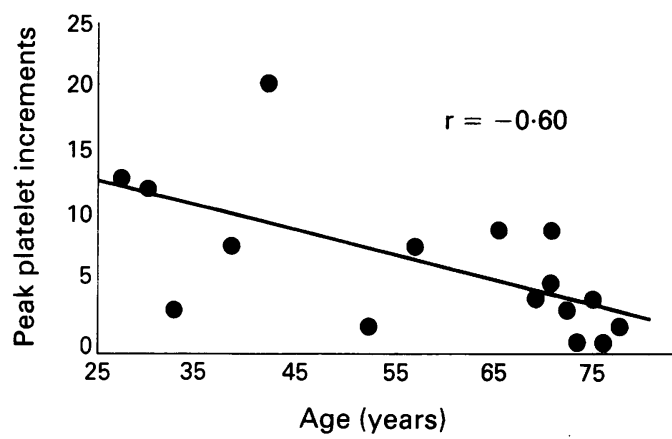

Figure 3 Patient No 16. Time course of response to ultrasound and sham resistant ITP. treatment in a patient with

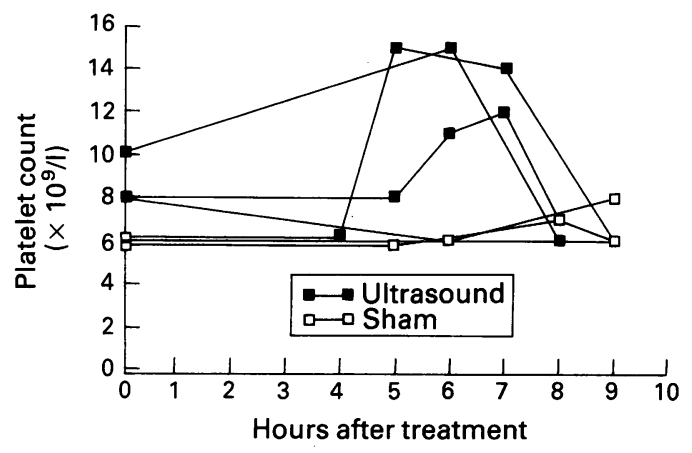

We were unable to show a positive doseresponse effect in this study but the range of doses used was limited; nor could we show any correlation between baseline platelet count and peak platelet increment. There was, however, a significant inverse correlation between patient age in the ITP-US group and peak platelet increment $(r=-0.60 ; \mathrm{p}=$ 0.015 ; fig 2).

Case 16 had resistant ITP and had failed to respond to prednisone, azathioprine, intravenous immunoglobulin, dapsone, and vincristine. She responded transiently to ultrasound treatment on three successive occasions with a platelet increment of 5,9 , and $4 \times 10^{9} / 1$ and a mean baseline platelet count of $7 \times 10^{9} / 1$ (fig 3 ). The effect was shortlived with onset at four to five hours, a peak at six hours, and a return to baseline by eight hours. There was no response to sham treatment on three occasions. Unfortunately she developed severe Staphylococcus aureus septicaemia after which she had an intracerebral haemorrhage and died. Spleen histology was consistent with ITP and there was no evidence to suggest splenic tissue damage from the ultrasound at postmortem examination.

Case 6 had ITP that failed to respond to steroids and vincristine and in whom there was no discernible platelet increase after ultrasound treatment. There was a moderate response to intravenous immunoglobulin with a rise in her platelet count from 20 to

Table 3 Group details

\begin{tabular}{llllll}
\hline Group & $n$ & $\begin{array}{l}\text { M:F } \\
\text { ratio }\end{array}$ & $\begin{array}{l}\text { Mean } \\
\text { age }(y)\end{array}$ & $\begin{array}{l}\text { Spleen } \\
\text { diameter (cm) }\end{array}$ & $\begin{array}{l}\text { Mean dose } \\
(\text { min })\end{array}$ \\
\hline ITP-US+NHL-US & 14 & $5: 9$ & 56 & $9 \cdot 0$ & $6 \cdot 8$ \\
ITP-SHAM & 9 & $4: 5$ & 63 & $9 \cdot 5$ & 0 \\
CONTROL-US & 5 & $3: 2$ & 29 & $8 \cdot 4$ & $6 \cdot 0$ \\
\hline
\end{tabular}

$\mathrm{n}=$ Number of subjects.
$70 \times 10^{9} / 1$. The spleen was removed during this time but it did not affect her platelet count, suggesting that in her case the spleen was of limited importance in mediating platelet destruction.

\section{Discussion}

Our results indicate that a single application of splenic ultrasound in patients with ITP results in a small but significant increase in platelet count over the ensuing four to eight hours. The effect was not seen in healthy controls or in sham treated patients. This supports the hypothesis that the platelet count in patients with ITP can be changed using a non-invasive physical modality such as ultrasound.

The mechanism of action of ultrasound is not completely understood. At high dose intensities (greater than $1.2 \mathrm{~W} / \mathrm{cm}^{2}$ ), it results in a significant thermal effect that may cause a local increase in metabolic rate and tissue perfusion. At very high dose intensities, it may even cause thermal necrosis. ${ }^{13} 14$ The dose of ultrasound used in our study $\left(1.0 \mathrm{~W} / \mathrm{cm}^{2}\right)$ caused a mild thermal effect at the skin surface-where the ultrasound exposure is greatest. Heating of the spleen could cause increased platelet sequestration secondary to increased blood flow and increased splenic macrophage metabolic activity. We did not find a decrease in platelet count, however, in any of our patients with ITP after ultrasound.

Ultrasound waves at subthermal intensities result in the transmission of pressure waves through the tissues, which in turn may result in the formation of $\mu \mathrm{m}$ sized bubbles. ${ }^{15}$ Fluid streams around these cavities disrupting cellular and subcellular function. ${ }^{16}$ Cell membranes are most susceptible to this effect as shown by altered transmembrane ionic flux and electrical potential. ${ }^{217}$ Altered membrane function of macrophages may be responsible for the beneficial effects of therapeutic ultrasound in the treatment of inflammatory lesions. ${ }^{1}$ A similar disruption of splenic macrophage function may mediate the rise in platelet count as macrophages play a pivotal part in the sequestration and destruction of platelets in ITP.

The time course of the response is interesting as little increment was seen before four hours. This supports the view that there may be more than one mechanism resulting in these unusual kinetics. The platelet count returned to baseline within nine hours in all cases, which suggests an effect on metabolically active cells (such as splenic macrophages in ITP) rather than some other longer term process such as the rate of platelet antibody production or cell replication.

Interestingly, several studies have identified increasing age of patients as a predictor of poorer response to spleen removal in ITP..$^{18-21}$ Our finding of a reduced response to ultrasound in older patients is in keeping with this phenomenon. The only patient in our study, case 6 , who failed to respond to spleen removal, also had a poor response to splenic 
ultrasound. These data suggest that response to splenic ultrasound may predict the response to splenectomy in ITP and further studies are in process to examine this point.

It may be possible to achieve a higher platelet increment by varying the dose intensity or duration of treatment. It would be of interest to know whether the platelet increment would increase or decrease with repeated ultrasound treatments. Case 16 was the only patient who received repeated treatments (three treatments on alternate days), and she showed no significant variation in response. There is no evidence from this study that the dose of ultrasound was harmful. Ultrasound has been applied by others in similar doses to the chest and abdomen without a single report of serious adverse effect. $^{22-26}$ The doses of ultrasound in our study were determined by the safety limits used by physiotherapists. Little is known of the toxicity of ultrasound at higher doses and careful evaluation of toxicity is required before more intensive dose schedules could be justified. Higher doses may ultimately be found to be safe and may result in clinically meaningful platelet increments.

We tested a single ultrasound frequency $(1 \mathrm{MHz})$ in this study, and other frequencies could result in higher platelet increments. The depth of the spleen below the skin is such, however, that ultrasound frequencies greater than $1.5 \mathrm{MHz}$ will not reach it effectively; and waves with frequencies less than $0.5 \mathrm{MHz}$ will penetrate the organ without being absorbed effectively. Also, the spleen varies in position from subject to subject and it moves with respiration. Furthermore, as soft tissue-air interfaces are virtually impermeable to ultrasound, the presence of the lower lobe of the left lung precludes access to the upper part of the organ. Despite these geometrical constraints, we are confident that the greater bulk of the tissue of the spleen was exposed to ultrasound although the exact dose delivered was impossible to determine.

To our knowledge, this is the first study which shows that ultrasound can be used to modulate the platelet count, albeit transiently, in patients with ITP. Further evaluation of the toxicity of ultrasound is advised before higher dose schedules are contemplated.

We thank ENRAF Nonius for the supply of an ultrasound machine and Jeffrey Hart, Anatomy Department, Guy's Hospital, for calibration of equipment. We are grateful to Dr
Norman Parker and Yvonne Watkins, Whittington Hospital, and Dr John England, Watford General Hospital, for helpful discussion.

1 Dyson $M$. Role of ultrasound in wound healing. In: Kloth LC, McCulloch JM, Feedare JA, eds. Wound healing: alternatives in management. Philadelphia: FA Davis, 1990:259-85.

2 Dinno MA, Dyson M, Young SR, Mortimer AJ, Hart J Crum LA. The significance of membrane changes in the safe and effective use of therapeutic and diagnostic ultrasound. Phys Med Biol 1989;34:1543-52.

3 Nyborg WL. Physical mechanisms for biological effects of ultrasound. Washington DC: HEW, 1977. (HEW publication (FDA) 7808062 .

4 Repacholi MH, Woodcock JP, Newman DL, Taylor KJW. Interaction of low intensity ultrasound and ionizing radiation with tumour cell surfaces. Phys Med Biol 1971;12:145-50.

5 Taylor KJW, Newman D. Electrophoretic mobility of Ehrlich cell suspensions exposed to ultrasound of varyEhrlich cell suspensions exposed to ultrasound

6 Rohr KR, Rooney JA. Effect of ultrasound on a bilaye Rohr KR, Rooney JA. Effect of ultrasound
lipid membrane. Biophys $₹$ 1978;23:33-40.

7 Berthold F, Berthold R, Matter I, et al. Effects of spleen exposure to ultrasound on cellular and antibodymediated immune reactions in man. Immunobiology 1982;162:46-55.

8 Anderson DW, Barrett JT. Depression of phagocytosis by ultrasound. Ultrasound Med Biol 1981;7:267-73.

9 Saad AH, Williams AR. Effects of therapeutic ultrasound and the clearance rate of blood borne colloidal particles in vivo. Br ₹ Cancer 1982;45:202-5.

10 Young SR, Dyson M. Macrophage responsiveness to therapeutic ultrasound. Ultrasound Med Biol 1990;16: apeutic

11 Saad AH, Williams AR. Effects of therapeutic ultrasound on the activity of the mononuclear phagocyte system in vivo. Ultrasound Med Biol 1986;12:145-50.

12 Young SR, Dyson M. Effect of therapeutic ultrasound on angiogenesis. Ultrasound Med Biol 1990;16:261-9.

13 Kumar RP, Ragu GS. Ultrasound induced damages and time bound recovery in mouse liver. Indian 7 Exp Biol 1989;27:23-5.

14 Colacchio TA, Coughlin C, Taylor J, Douple E, Ryan T, Crichlow RW. Intraoperative radiation therapy and hyperthermia. Arch Surg 1990;125:370-5.

15 Ter Haar GR, Daniels S. Evidence of ultrasonically induced cavitation in vivo. Phys Med Biol 1981;26: 1145-9.

16 Dyson $M$. Therapeutic applications of ultrasound. In Nuborg WL, Ziskin MC, eds. Biological effects of ultrasound. Edinburgh: Churchill-Livingstone, 1985:121-34.

17 Mortimer AJ, Dyson M. The effect of therapeutic ultrasound on calcium uptake in fibroblasts. Ultrasound Med Biology 1988;14:499-506.

18 Di Fino SM, Lachant MA, Kirshner JJ, Gottlieb AJ. Adul idiopathic thrombocytopenic purpura. Clinical response to therapy. Am $\mathcal{J}$ Med 1980;69:430-42.

19 Akwari OE, Itani KMF, Coleman RE, Rosse WF. Splenectomy for primary and recurrent immune thrombocytopenic purpura (ITP): current criteria for patien selection and results. Ann Surg 1987;206:529-41.

20 Fenaux P, Caulier MT, Hirschauer J, Beuscart $R$, Goudemand J, Bauters F. Reevaluation of the prognostic factors for splenectomy in chronic idiopathic thrombocytopenic purpura (ITP). Eur $\mathcal{F}$ Haematol 1989;42 $259-64$.

21 Fabris F, Zanatta N, Casonato A, Randi ML, Luzzato G, Girolami A. Responses to splenectomy in idiopathic thrombocytopenic purpura: prognostic value of the clini$\mathrm{cal}$ and laboratory evaluation. Acta Haematol 1989; 81:28-33.

22 Garrett AS, Garrett M. Ultrasound therapy for herpes zoster pain. $\mathcal{F} R$ Coll Gen Pract 1982;11:709-10.

23 Nwuga VCB. Ultrasound in the treatment of back pain resulting from prolapsed intervertebral disc. Arch Phys Med Rehabil 1983;64:88-9.

24 Jones RJ. Treatment of acute herpes zoster using ultrasonic therapy. Physiotherapy 1984;70:96-7.

25 Payne C. Ultrasound for post-herpetic neuralgia. Physiotherapy 1984;70:96-7.

26 Levenson JL, Weissberg MP. Ultrasound abuse: A case report. Arch Phys Med Rehabil 1983;64:90-1. 